\title{
Path planning of mobile robot based on multi-sensor information fusion
}

\author{
Ruixia Xu
}

\begin{abstract}
Mobile robot is a very important branch of robotics. In practice, the performance of mobile robot is required to be higher and higher. It is required that mobile robot can adapt to different complex environments through its own intelligent system to achieve the established functional goals. Compared with traditional path planning with single sensor information, Kalman filter is used to fuse multi-sensor information, and path planning method based on improved dynamic artificial potential field method is studied. Using the improved dynamic artificial potential field method, the robot can also achieve optimal path planning and obstacle avoidance in complex dynamic environment. The simulation results show that the proposed algorithm is feasible.
\end{abstract}

Keywords: Mobile robot, Multi-sensor, Information fusion, Path planning, Artificial potential field method

\section{Introduction}

The development of computer technology promotes the progress of intelligent robots. Modern intelligent robots can plan and make decisions through different kinds of sensors, so as to achieve different functional goals [1]. Intelligent robots have been used in various industries. The birth of this new intelligent tool has constantly changed the traditional production mode of people, which greatly improving human productivity and reducing human production risks [2]. Among these robots, mobile robots are widely used. Mobile robots use sensors to identify the environment and locate their own positions [3]. The sensors of the robot can be divided into internal and external sensors. The external sensors include laser range finder, fiber optic sensor, and ultrasonic sensor. Internal sensors include gyroscopes and odometers. [4]. However, there is a problem of inaccurate information in the process of data acquisition for sensors in mobile robots. The environment of mobile robots is also a dynamic environment, and it is also a moving object itself. Therefore, the traditional sensor technology of mobile robots has been difficult to meet the accuracy and stability requirements of mobile robots [5]. Foreign robotics and computer technology development earlier in Japan, Europe, and other developed countries on mobile robot research and production has

Correspondence: xrxsdp@126.com

Shandong Polytechnic, Jinan, Shandong, China been at the international advanced level. Domestic robot research and development later, after the reform and opening up, China's research on robotics has made great progress, but after all, it started too late.

\section{State of the art}

Information fusion technology, like traditional high tech, originates from the military field, which originated from the American Navy in the 1970s [6]. After the development in the 1980s and 1990s, the information fusion technology applied in the military has been extended to many civil fields such as electronic commerce, medical diagnosis, and wireless communication. Compared with traditional single-sensor technology, multi-sensor information fusion has the following advantages: improving system reliability, enhancing system robustness, reliability and detection performance, improving data accuracy, reducing system cost, and wider space-time coverage of the system [7]. However, the existing theory of how far information fusion cannot meet the needs of modern mobile robot systems. At the same time, a very important function and capability requirement of mobile robot is navigation and positioning, so as to realize the path planning of mobile robot [8]. Robot positioning is the need of a suitable and effective algorithm, so that the robot can accurately judge the environmental laws and their location, so as to achieve the obstacle avoidance walk and reach the destination [9]. At present, the most 
widely used localization algorithms are internal sensor localization, vision sensor localization, active-sensor localization, laser-scanning localization, and GPS localization. The path planning of mobile robot mainly solves four problems: coordination strategy, environment expression, path execution, and planning method [10]. There are many ways and classifications of path planning, which can be divided into centralized and decentralized planning according to the planners, online and offline planning according to the planning time, and local and global path planning according to the known environment. In order to meet the increasing demands of mobile robots in China, it is very important to study the path planning of multi-sensor information fusion technology [11].

\section{Methodology}

\subsection{Multi-sensor information fusion technology}

The path planning of mobile robot is to locate the robot accurately. Generally speaking, the environment of the robot is regarded as a two-dimensional plane, and the sensor used by the robot determines its location mode [12]. The robot's positioning mode is determined by the sensors it uses, such as odometer, gyroscope, and laser radar. The cumulative error of odometer positioning sensor is larger, which will be affected by body skidding, wheel deformation, load changes, and other errors, and this error will increase with the increase of moving distance. The Kalman filter is used to fuse sensor information to reduce error [13]. The tracked mobile robot is selected for research, and its posture diagram is shown in Fig. 1. It adopts the Cartesian coordinate system of XOY. The robot body local coordinate system is $x c y$, the robot front is the $y$ axis, the $x, y$ axis between the angle is $\theta$, thus it can get the robot pose vector:

$$
p=(x, y, \theta)^{T}
$$

The robot's vehicle body and track width are $L, b$, the robot revolver and the right wheel are $v_{L}, v_{R}$ respectively, thus the kinematics model formula of robots can be obtained:

$$
\dot{p}=\left(\begin{array}{c}
\dot{x} \\
\dot{y} \\
\dot{\theta}
\end{array}\right)=\left(\begin{array}{ccc}
\frac{1}{2} \cos \theta & \frac{1}{2} \cos \theta \\
\frac{1}{2} \sin \theta & \frac{1}{2} \sin \theta \\
-\frac{1}{L+2 b} & \frac{1}{L+2 b}
\end{array}\right)\left(\begin{array}{l}
v_{L} \\
v_{R}
\end{array}\right)
$$

The linear speed and angular velocity of the robot are $v, w$ respectively, and their relationship with the speed of the robot wheels is:

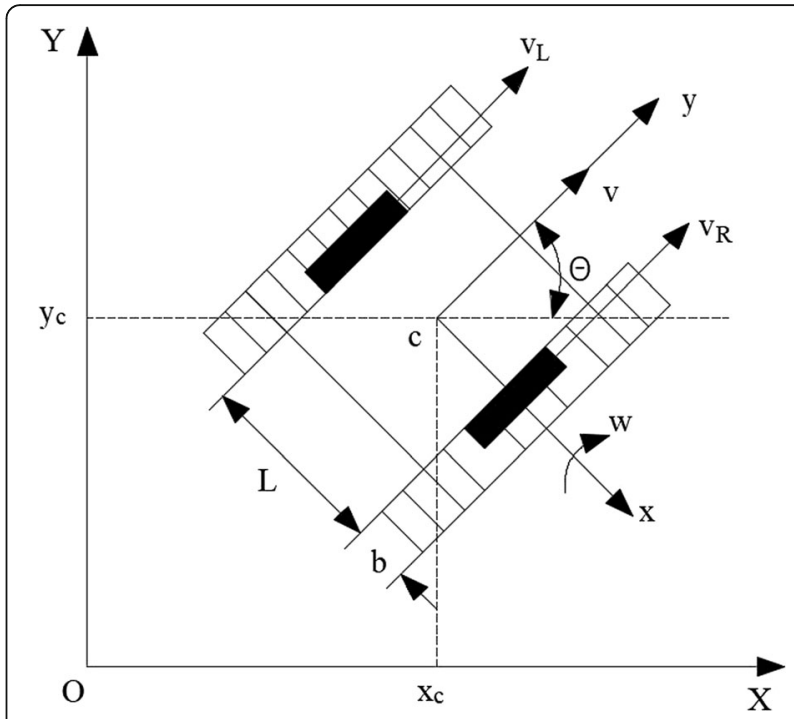

Fig. 1 Position and attitude diagram of tracked mobile robot

$$
\left(\begin{array}{c}
v \\
w
\end{array}\right)=\left(\begin{array}{cc}
\frac{1}{2} & \frac{1}{2} \\
-\frac{1}{L+2 b} & \frac{1}{L+2 b}
\end{array}\right)\left(\begin{array}{l}
v_{L} \\
v_{R}
\end{array}\right)
$$

The combined formula (2) can be used:

$$
\dot{p}=\left(\begin{array}{c}
\dot{x} \\
\dot{y} \\
\dot{\theta}
\end{array}\right)=\left(\begin{array}{cc}
\cos \theta & 0 \\
\sin \theta & 0 \\
0 & 1
\end{array}\right)\left(\begin{array}{l}
v \\
w
\end{array}\right)
$$

The turning radius of the robot is $R$ :

$$
R=\frac{v}{w}=\frac{L+2 b}{2} \cdot \frac{v_{L}+v_{R}}{-v_{L}+v_{R}}
$$

It can be seen from the formula that the motion state of the robot is different when the speed of the left wheel and the right wheel is different. (1) If $v_{L}=-v_{R}, R=0$, the robot is moving around itself. (2) If $v_{L}=v_{R}, R \rightarrow \infty$, it shows that the robot is moving in a straight line. (3) If $v_{L} \neq v_{R}$, it indicates that the robot is circumferential motion with radius $R$.

According to the existing research, the combination of predictive positioning and Kalman filter differential acoustic positioning can effectively reduce the cumulative error of sensor components and, thus, improve the positioning performance of the robot. According to the fusion algorithm of Kalman filter, the position of $K$ time is expressed as:

$$
p(k)=[x(k) y(k) \theta(k)]^{T}
$$

Formula (6) shows that the optimal position of the robot is estimated to be $\hat{p}(k \mid k)$, and the robot is driven to the position of $p(k+1)$ by controlling the input $u(k)$. Then, at $(k+1)$, there is: 


$$
\begin{aligned}
\hat{p}(k+1 \mid k)= & \hat{p}(k \mid k)+u(k) \\
= & \hat{p}(k \mid k) \\
& +\left[\begin{array}{l}
\frac{\Delta S_{r}+\Delta S_{l}}{2} \cos \left(\theta+\frac{\Delta S_{r}-\Delta S_{l}}{2 b}\right) \\
\frac{\Delta S_{r}+\Delta S_{l}}{2} \sin \left(\theta+\frac{\Delta S_{r}-\Delta S_{l}}{2 b}\right) \\
\frac{\Delta S_{r}-\Delta S_{l}}{b}
\end{array}\right]
\end{aligned}
$$

The updated covariance matrix is further obtained, specifically:

$$
\Sigma_{p}(k+1 \mid k)=\nabla_{p} f \cdot \Sigma_{p}(k \mid k) \cdot \nabla_{p} f^{T}+\nabla_{p} f \cdot \Sigma_{u}(k \mid k) \cdot \nabla_{u} f^{T}
$$

In formula (8),

$$
\Sigma_{u}=\operatorname{cov}\left(\Delta S_{r}, \Delta S_{l}\right)=\left[\begin{array}{cc}
k_{r}\left|\Delta S_{r}\right| & 0 \\
0 & k_{l}\left|\Delta S_{l}\right|
\end{array}\right]
$$

From the predicted robot position and the corresponding measurement equation, the measurement and prediction can be calculated.

$$
\hat{z}_{i}(k+1)=h_{i}\left(z_{t, i}, \hat{p}(k+1 \mid k)\right)
$$

According to the observation and position prediction of the mobile robot at $k+1$ time, the best position estimation $\hat{p}(k+1 \mid k+1)$ of the robot can be calculated by using the updated weight coefficient $S(k)$.

$$
K(k+1)=P(k+1 \mid k) H(k+1)^{T} S(k+1)^{-1}
$$

The state update equation is:

$$
\begin{aligned}
\hat{x}(k+1 \mid k+1)= & \hat{x}(k+1 \mid k) \\
& +K(k+1) \gamma(k+1)
\end{aligned}
$$

The covariance update equation is:

$$
P(k+1 \mid k+1)=P(k+1 \mid k)-K(k+1) S(k+1)^{-1}
$$

The setting of the data model is done by inputting variables and measuring variables, which is generally Gaussian white noise. If the input and measurement covariance information are known, then the state of the robot system at different sampling times can be calculated through these information data, and finally the positioning results can be calculated. The frame diagram of location method based on Kalman filter and information technology is shown in Fig. 2.

\section{Research on path planning algorithm}

Robot path planning is the key technology of mobile robot, it is able to ensure that the robot and the surrounding environment and obstacles do not collide, a good path planning can also be achieved according to established indicators to achieve optimal path. Commonly used path planning methods are A * algorithm, C-space method, artificial potential field method and so on. Considering the fact that the environment in which the robot is located is not a static environment, but a dynamic environment, the improved dynamic artificial potential field method is selected to construct the potential field function of the mobile robot.

The artificial potential field method is proposed by Dr. Khatib in 1985. It is originally proposed to enable the manipulator to have obstacle avoidance function. Artificial potential field method (APF) is widely used in robot path planning because of its advantages such as less computation, simple structure, and easy control at the bottom. Artificial potential field method is to introduce the concept of physical field into the expression of planning environment. Its essential idea is to describe the spatial structure through the numerical function of artificial potential field, and the force traction robot in the potential field achieves the goal. Using the potential field method to study the path planning of mobile robot is to regard the robot as a point under the influence of the field potential. The robot moves along the field like a ball rolling down a mountain. In the presence of potential, obstacles represent repulsion, and the smallest value in space is gravity. In the configuration space, the robot is regarded as a point. Under the traction of the artificial field potential, the robot can avoid obstacles in the path and move smoothly to the target point.

Artificial potential field method is not only a path planning, but also a field generated by the control vector of the robot. If the robot uses the sensor installed in its own sensor, it can locate in the potential field and judge the robot's motion attitude at the next moment. In conclusion, the essential idea of the potential field method is to be attracted by the target and rejected by the detected obstacles at the same time. In the course of traveling, when obstacles appear in the path detected by the robot, the original potential field will be updated, and the corresponding potential field force will change, thus changing its path. Robot repulsion potential field is to produce a force far away from the detected obstacles, the repulsion force will be stronger with the robot and obstacles closer, and when the robot and obstacles are far away, the influence will become smaller and smaller.

However, the artificial potential field method has some relative disadvantages: It is difficult to find the path when the obstacles are close to each other, the field potential balance will fall into a local minimum and swing in a narrow channel. Potential field method has been perfected continuously, and the most popular one is to solve the problem of minimum in potential 


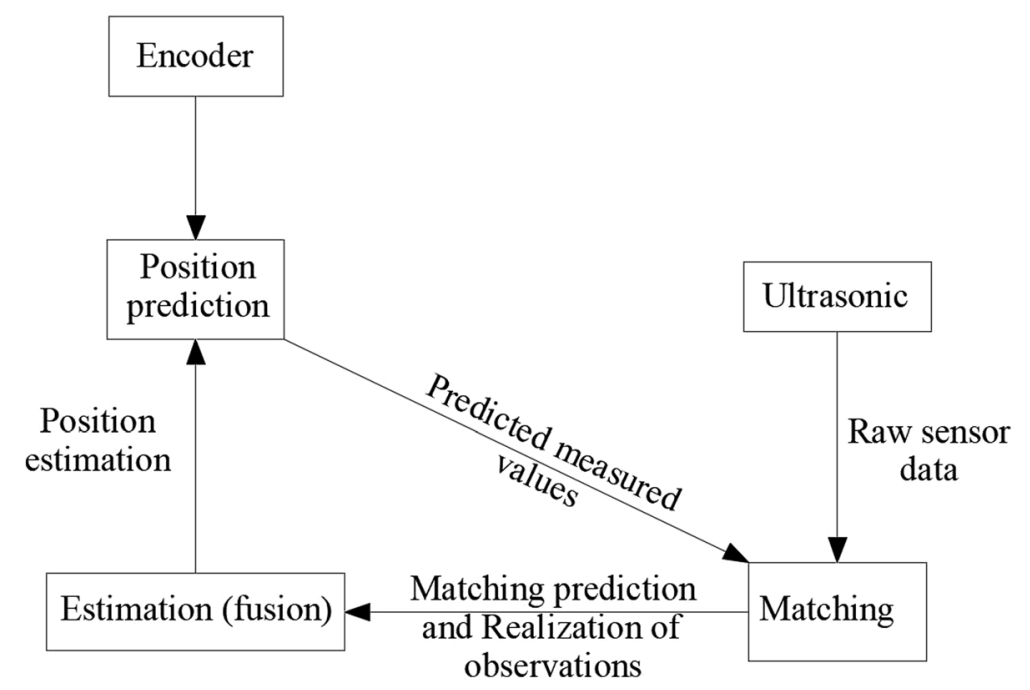

Fig. 2 Kalman filter positioning framework for robots

field method. At present, many methods of jumping out of local minimum have been developed, such as wall-walking, escape, simulated annealing, and other methods that are more effective.

Figure 3 is the velocity decomposition of the robot in the gravitational field and its motion state. Let the robot speed and the target point speed at $t$ moment be $V_{n} V_{g}$ respectively. After decomposing the robot speed, one of them is the velocity vector relative to the target point and the other is the same as the velocity direction of the target point. The formula is as follows:

$$
\begin{aligned}
& V_{r g}=V_{r}-V_{g} \\
& \phi_{1}=\angle V_{r}-\angle V_{g}
\end{aligned}
$$

The formula of the gravitational field function can be obtained as follows:

$$
U_{a t t v}=\frac{K_{a t t v}\left(V_{r}-V_{g}\right)^{2}}{2}
$$

Pair formula (16) calculates negative gradient:

$$
\begin{aligned}
& F_{a t t v}=-\nabla U_{a t t v}=K_{a t t v}\left(V_{r}-V_{g}\right) \\
& F_{a t t}=F_{a t t v}+F_{a t t p}
\end{aligned}
$$

Then, can calculate:

$$
F_{a t t v}=K_{a t t v}\left[\left(V_{r}-V_{g}\right) \cos \phi_{1} x+\left(V_{r}+V_{g}\right) \sin \phi_{1} y\right]
$$

In the gravitational field, the direction of the robot pointing at the target position is the direction of the position gravitational force $F_{a t t p}$, whose magnitude is proportional to the distance relative to the target position. Correspondingly, the direction of velocity gravity $F_{a t t v}$ is relative to the direction of motion of the robot. It can keep the speed of the robot and the target the same. When the relative speed is zero gravity, it will return to zero.

The repulsive field model based on the repulsive velocity function is further introduced. The motion state of the robot in the repulsive field is shown in Fig. 3. Let the velocity relative to the obstacle of the robot be $V_{o n}$ the velocity vector of the obstacle be $V_{o b s}$, the velocity
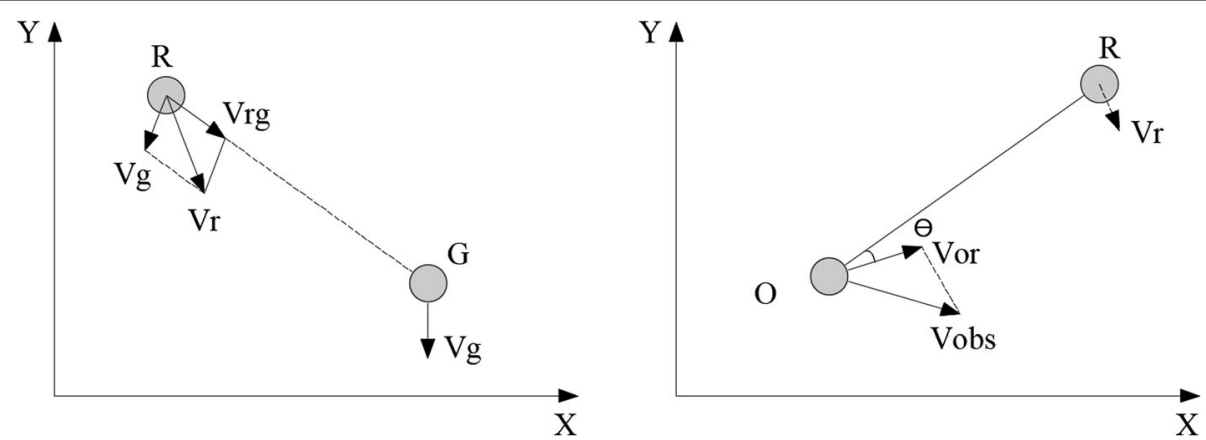

Fig. 3 Velocity decomposition of robot in gravitational field and its state of motion with obstacles 


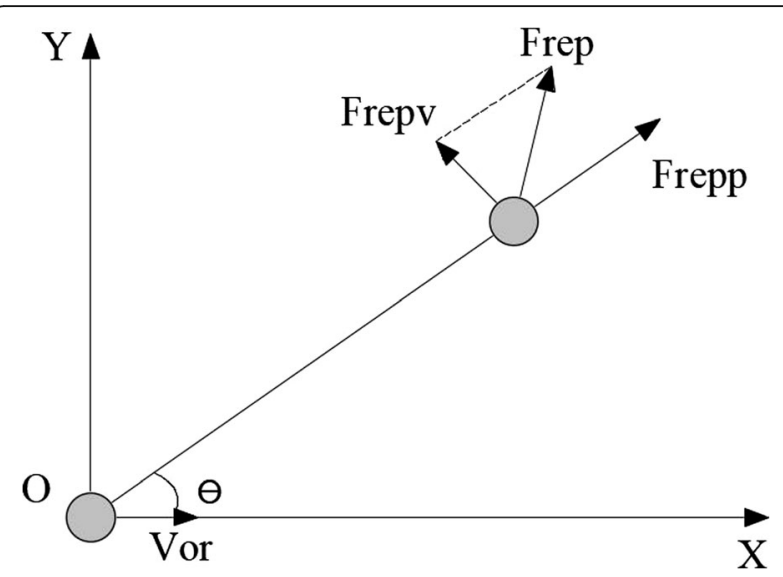

Fig. 4 Repulsive force diagram of robot field

repulsion coefficient be $K_{\text {repw }}$ the position vector of the robot relative to the obstacle and the angle between the velocity vector and the velocity vector be $\theta$, and the angle between $V_{o r}$ and the $x$ axis be $\phi_{2}$. The expression formula of repulsive potential field function is as follows:

$$
U_{\text {repv }}=K_{\text {repv }}\left\|V_{\text {obs }}-V_{r}\right\| \sin \theta=K_{\text {repv }} V_{\text {or }} \sin \theta
$$

To build the coordinate system, as shown in Fig. 4, the robot coordinates are $\mathrm{XC}$ relative to the obstacle, thus the repulsion field function formula of the obstacle can be obtained:

$$
U_{\text {repv }}=K_{\text {repv }} V_{o r} \sin \theta=K_{\text {repv }} V_{o r} \frac{Y_{o} r}{\sqrt{X_{o r}^{2}+Y_{o r}^{2}}}
$$

The formula of velocity repulsion function can be obtained by calculating the position of velocity repulsion field:

$$
F_{\text {repv }}=\frac{K_{\text {repv }} V_{o r}}{\sqrt{X_{o r}^{2}+Y_{o r}^{2}}}\left(-\sin \theta \cos \phi_{2} x+\cos \theta \sin \phi_{2} y\right)
$$

If the robot is not in the scope of the repulsive force field, the motion of the robot will not be affected by the obstacle when the distance between the robot and the obstacle is very long, so the repulsive force of the robot is 0 . On the other hand, if $\cos \theta<0$, then the robot is moving away from the obstacles, repulsion will gradually reduce to 0 , which is also considered to be the relative speed of the repulsion of 0 . So, there are:

$$
F_{\text {rep }}=\left\{\begin{array}{l}
K_{\text {repv }} V_{o r} \\
\sqrt{X_{o r}^{2}+Y_{o r}^{2}}
\end{array}\left(-\sin \theta \cos \phi_{2} x+\cos \theta \sin \phi_{2} y\right), \theta \in\left(-\frac{\pi}{2}, \frac{\pi}{2}\right), d_{o r} \leq d_{o}\right.
$$

\section{Result analysis and discussion}

In order to verify the path planning technology of multi-sensor information fusion based on Kalman filter, the tracked intelligent mobile robot is applied to the research. This type of mobile robot adopts DC servo control with high motion precision and high load capacity. The assembled sensors include ultrasonic, visual, and compass sensors. It can use notebook computer or PCI motion controller. It has the characteristics of high intelligence and multi-module. The performance parameters of the mobile robot are shown in Table 1:

The mobile robot adopts modular design, so it can formulate and implement its own tasks according to different needs of users. Because of the open design, it is highly scalable, and users can directly manipulate and analyze the bottom layer of the control system of the mobile robot. In addition, the mobile robot has a wealth of supporting software, including communication software modules, image acquisition, and processing toolkits, and is also compatible with third-party software systems.

In this experiment, the length and width of the test site are $4.5 \mathrm{~m}$ and $3.5 \mathrm{~m}$ respectively. Wood blocks and tables and chairs are used as obstacles for the test. Because of the limited experimental environment, static obstacles are only used for verification. In actual environment, the obstacle avoidance control structure diagram of robot is shown in Fig. 5. All the algorithms are downloaded to the control computer and communicated

Table 1 Robot performance parameter list

\begin{tabular}{llllll}
\hline Number & Parameter class & Technical parameter & Number & Parameter class & Technical parameter \\
\hline 1 & Size & $L \times \mathrm{D} \times \mathrm{H}: 900 \mathrm{~mm} \times 600 \mathrm{~mm} \times 420 \mathrm{~mm}$ & 9 & Maximum climbing height & $100 \mathrm{~mm}$ \\
2 & Material science & Aluminum alloy & 10 & Maximum cross-country height & $350 \mathrm{~mm}$ \\
3 & Weight & $100 \mathrm{~kg}$ & 11 & Maximum climbing slope & $30^{\circ}$ \\
4 & Chassis height & $55 \mathrm{~mm}$ & 12 & Battery capacity & $24 \mathrm{~V} 48 \mathrm{Ah}$ \\
5 & Deceleration ratio & $1: 32$ & 13 & Charging time & $10 \mathrm{~h}$ \\
6 & Allowable minimum & $10 \mathrm{~mm} / \mathrm{s}$ & 14 & Operating system & Windows2000 \\
7 & advance speed & & 15 & Development tool & VC++ \\
8 & Effective load & $30 \mathrm{~kg}$ & 16 & In-place gyration radius & $300 \mathrm{~mm}$ \\
\hline
\end{tabular}




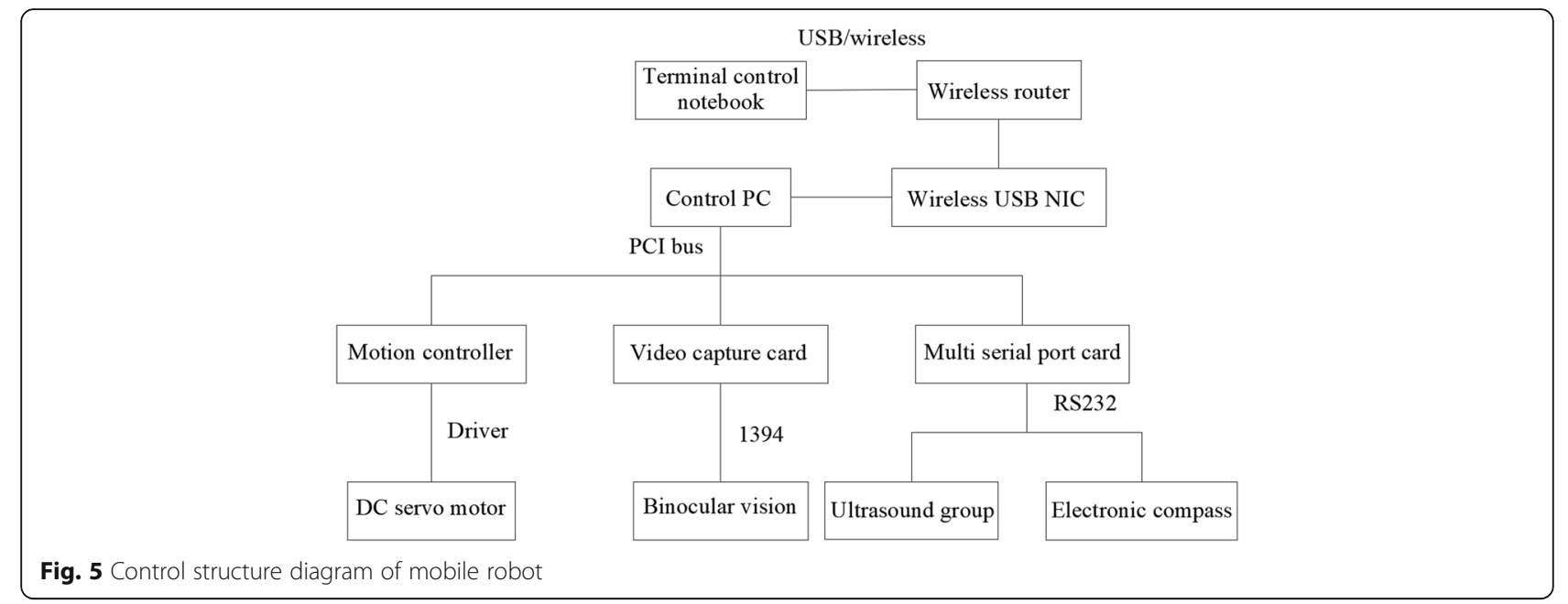

via wireless network card and wireless router. The protocol is $54 \mathrm{M}$ broadband shift and $202.22 \mathrm{~g}$ wireless communication protocol. The feedback information of ultrasonic sensor is the input through RS232 serial port, and the information received by robot is the output by pulse through motion controller. Finally, the speed of robot is controlled by DC servo driver.

Set the initial position of the robot to be $(0,0)$, the target position to be $(400,70)$, the initial speed of the robot to be set to $0 \mathrm{~m} / \mathrm{s}$, and the initial attitude angle to be 0 rad. Due to the limited space in the experimental site, the maximum speed of the robot is limited to $0.1 \mathrm{~m} / \mathrm{s}$ in order to avoid too fast and not easy to operate. The position change curve of the robot is shown in Fig. 6.

The velocity and attitude angle curves of the robot are shown in Fig. 7.

When the robot detects indoor obstacles, it will take the initiative to avoid obstacles. When the robot runs within the repulsive force range of the obstacle, the robot can detect the obstacle in the laboratory through the sensor. The robot uses the path planning algorithm to calculate the potential force and bypass the obstacle and redesigns a more reasonable optimal path. Even if the robot is in a relatively complex environment, the
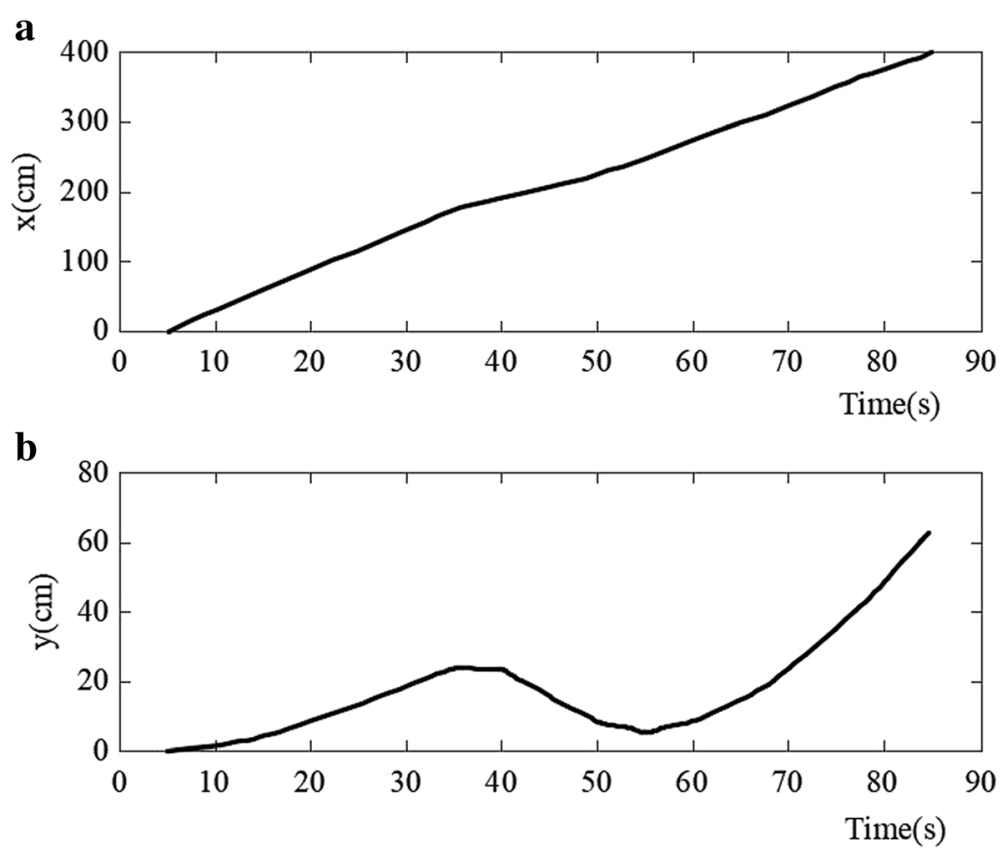

Fig. 6 Robot position change curve $\mathbf{a} \& \mathbf{b}$ 

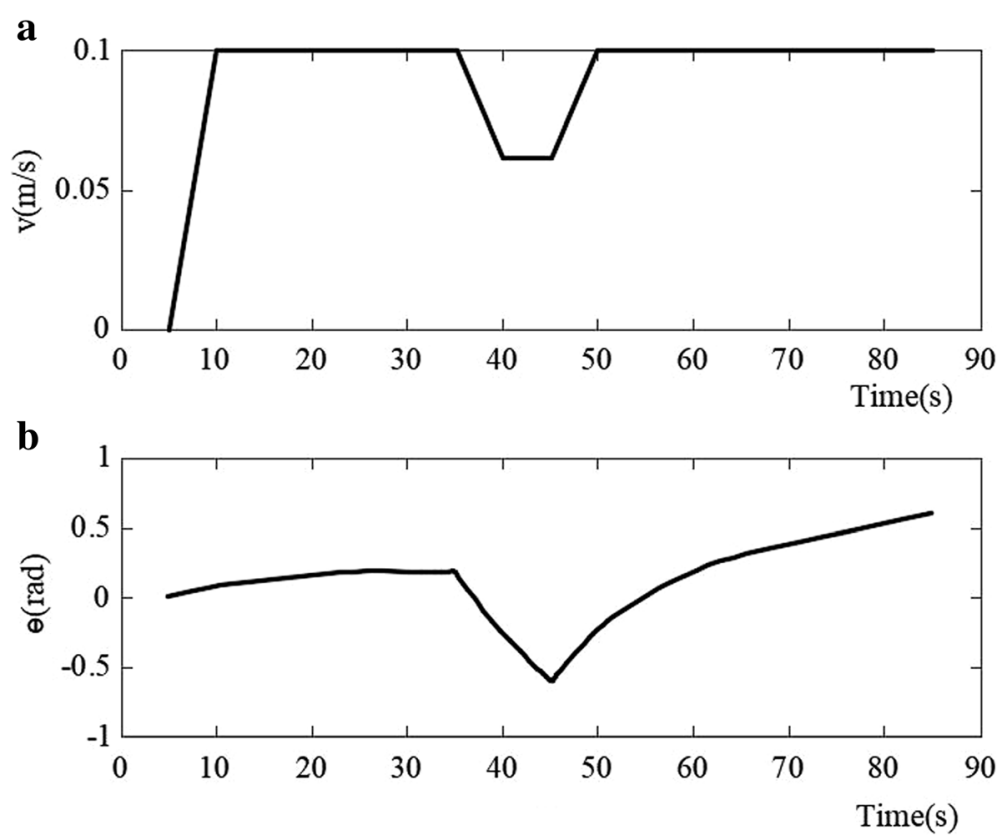

Fig. 7 a, b Curve of speed and attitude angle of robot

robot can improve the potential field function, so as to calculate the resultant force and through the narrow space, will not fall into the local minimum, and eventually successfully reach the set goal point. This proves that the improved artificial potential field method has a good application effect and can realize the intelligent path planning of mobile robot.

\section{Conclusion}

The development of mobile robot technology shows the scientific and technological levels of a country to a certain extent. Although the research of mobile robot in China has made a lot of achievements in recent years, there are still many technical problems. Aiming at the problems of traditional path planning methods for mobile robots, Kalman filter is used to fuse data and realize localization, which makes it possible for mobile robots to use this localization method to obtain higher localization accuracy. In order to better realize the path planning of mobile robot, the traditional artificial potential field method is introduced, and an improved dynamic artificial potential field method is proposed to overcome the shortcomings of the traditional artificial field potential method. In order to verify the proposed algorithm, the path planning effect of tracked mobile robot in static complex environment is tested by simulating in the laboratory of $4.5 \mathrm{~m} \times 3.5 \mathrm{~m}$. The simulation results show that the improved path planning algorithm can make the mobile robot avoid obstacles smoothly in complex and narrow space and realize the optimal path planning to achieve the set goal. Due to the limited experimental environment, the simulation experiment is only a simulation experiment in a complex static environment. There is no application research in an unknown complex dynamic environment, and further research is needed.

\section{Funding \\ No.}

Availability of data and materials

Data sharing not applicable to this article as no datasets were generated or analysed during the current study.

Author's contributions

RX has made a lot of contributions to path planning of mobile robot based on multi-sensor information fusion, and I also handled the sorting of the article. The author read and approved the final manuscript.

\section{Competing interests}

The author declares that she has no competing interests.

\section{Publisher's Note}

Springer Nature remains neutral with regard to jurisdictional claims in published maps and institutional affiliations.

Received: 6 December 2018 Accepted: 11 January 2019

Published online: 21 February 2019

\section{References}

1. Z.X. Liu, C.X. Xie, M. Xie, et al., Mobile robot positioning method based on multi-sensor information fusion laser SLAM. Clust. Comput. 84, 1-7 (2018)

2. Á. Odry, R. Fullér, I.J. Rudas, et al., Kalman filter for mobile-robot attitude estimation: novel optimized and adaptive solutions. Mech. Syst. Sig. Process 110(C), 569-589 (2018)

3. Q. Qian, J. Wu, Z. Wang, A novel configuration of two-wheeled selfbalancing robot. Tehnicki Vjesnik 24(2), 459-464 (2017)

4. J. Yuan, J. Zhang, S. Ding, et al., Cooperative localization for disconnected sensor networks and a mobile robot in friendly environments. Inf. Fusion $37(C), 22-36(2017)$ 
5. H. Gao, Q. Wei, Y. Yu, et al., Mobile robot obstacle avoidance algorithms based on information fusion of vision and sonar. Int. J. Future Gener. Commun. Netw. 9(8), 111-120 (2016)

6. Y. Zhang, K. Xu, C. Zheng, et al., Advanced research on information perception technologies of intelligent electric vehicles. Chin. J. Sci. Instrum. 38(4), 794-805 (2017)

7. A. Ismail, M. Elmogy, H. Elbakry, Landmines detection using low-cost multisensory mobile robot. J. Convergence Inf. Technol. 10(6), 51-60 (2015)

8. A. Canedo-Rodríguez, V. Álvarez-Santos, C.V. Regueiro, et al., Particle filter robot localisation through robust fusion of laser, WiFi, compass, and a network of external cameras. Inf. Fusion 27, 170-188 (2016)

9. L.I. Xiu-Zhi, S.M. Jia, 3D map building for mobile robot based on multisensor fusion aided SLAM. Trans. Beijing Inst. Technol. 35(3), 262-267 (2015)

10. Y. Zhang, D.P. Hong, Navigation of mobile robot using low-cost GPS. Int. J. Precision Eng. Manuf. 16(4), 847-850 (2015)

11. M. Kumar, Y. Mao, Y. Wang, et al., Fuzzy theoretic approach to signals and systems: static systems. Inf. Sci. 418-419, 668-702 (2017)

12. W. Zhang, K. Thurow, R. Stoll, A context-aware mhealth system for online physiological monitoring in remote healthcare. Int. J. Comput. Commun. Control 11(1), 142-156 (2016)

13. H. Li, X. Li, X. Xu, et al., Modeling departure time choice of metro passengers with a smart corrected mixed logit model - a case study in Beijing. Transp. Policy 69, 106-121 (2018)

\section{Submit your manuscript to a SpringerOpen ${ }^{\circ}$ journal and benefit from:}

- Convenient online submission

- Rigorous peer review

- Open access: articles freely available online

- High visibility within the field

- Retaining the copyright to your article

Submit your next manuscript at $\boldsymbol{\nabla}$ springeropen.com 